\title{
An ancient spliceosomal intron in the ribosomal protein L7a gene (Rpl7a) of Giardia lamblia
}

\author{
Anthony G Russell ${ }^{\dagger}$, Timothy E Shutt ${ }^{\dagger}$, Russell F Watkins and \\ Michael W Gray*
}

Address: Program in Evolutionary Biology, Canadian Institute for Advanced Research, Department of Biochemistry and Molecular Biology, Dalhousie University, Halifax, Nova Scotia B3H 1X5, Canada

Email: Anthony G Russell - russella@dal.ca; Timothy E Shutt - tshutt@dal.ca; Russell F Watkins - rwatkins@dal.ca;

Michael W Gray* - m.w.gray@dal.ca

* Corresponding author †Equal contributors

Published: 18 August 2005

BMC Evolutionary Biology 2005, 5:45 doi:10.1/86/147|-2|48-5-45

This article is available from: http://www.biomedcentral.com/I47/-2/48/5/45

(C) 2005 Russell et al; licensee BioMed Central Ltd.

This is an Open Access article distributed under the terms of the Creative Commons Attribution License (http://creativecommons.org/licenses/by/2.0), which permits unrestricted use, distribution, and reproduction in any medium, provided the original work is properly cited.
Received: 22 April 2005

Accepted: 18 August 2005

\begin{abstract}
Background: Only one spliceosomal-type intron has previously been identified in the unicellular eukaryotic parasite, Giardia lamblia (a diplomonad). This intron is only 35 nucleotides in length and is unusual in possessing a non-canonical 5 ' intron boundary sequence, CT, instead of GT.

Results: We have identified a second spliceosomal-type intron in G. lamblia, in the ribosomal protein L7a gene (Rp/7a), that possesses a canonical GT 5' intron boundary sequence. A comparison of the two known Giardia intron sequences revealed extensive nucleotide identity at both the $5^{\prime}$ and $3^{\prime}$ intron boundaries, similar to the conserved sequence motifs recently identified at the boundaries of spliceosomal-type introns in Trichomonas vaginalis (a parabasalid). Based on these observations, we searched the partial $G$. lamblia genome sequence for these conserved features and identified a third spliceosomal intron, in an unassigned open reading frame. Our comprehensive analysis of the Rpl7a intron in other eukaryotic taxa demonstrates that it is evolutionarily conserved and is an ancient eukaryotic intron.
\end{abstract}

Conclusion: An analysis of the phylogenetic distribution and properties of the $R p / 7 a$ intron suggests its utility as a phylogenetic marker to evaluate particular eukaryotic groupings. Additionally, analysis of the $G$. lamblia introns has provided further insight into some of the conserved and unique features possessed by the recently identified spliceosomal introns in related organisms such as $T$. vaginalis and Carpediemonas membranifera.

\section{Background}

Spliceosomal introns have now been identified in all major eukaryotic lineages. Recently added to this list are several protists that are widely considered to represent deep divergences within Eucarya: the diplomonad Giardia lamblia [1], its close relative Carpediemonas membranifera [2], and the parabasalid Trichomonas vaginalis [3]. The dis- tribution and conservation of proteins involved in the removal of spliceosomal introns $[1,4,5]$ suggests that this intron type is a feature that was present in the ancestor of this domain of life. While the precise determination of intron frequency in the T. vaginalis and C. membranifera genomes awaits further analysis, these preliminary studies suggest that these two organisms likely contain many 
more spliceosomal introns than are evident in the compact genome of G. lamblia. To date, only one spliceosomal-type intron has been reported in G. lamblia despite the analysis of approximately 5800 predicted open reading frames (ORFs) [1].

One of the most interesting properties of the T. vaginalis introns is the presence of a functionally important motif and putative branch-point sequence, ACTAAC, invariantly located within a 'conserved' 12-nucleotide (nt) intron segment located directly adjacent to the 3 ' splice site. Surprisingly, the only known G. lamblia intron also contains the identical 12-nt motif at its $3^{\prime}$ end. Additionally, the introns from these two organisms display significant sequence similarity at their 5 ' ends. This suggests that similar splicing mechanisms may be employed to remove introns in organisms from these two eukaryotic groups, members of the proposed eukaryotic supergroup Excavata [6]. These sequence motifs have been shown to be important for efficient in vivo splicing of introns in $T$. vaginalis [3]; however, since only one intron has been identified in G. lamblia so far, the extent of conservation of these sequence motifs in other G. lamblia introns is not known. It is conceivable that these intron features are indicative of ancestral eukaryotic spliceosomal introns. Conversely, these features may be derived and thus be unique among spliceosomal introns found in these long-branching eukaryotic taxa.

It has been proposed that most of the identified T. vaginalis introns are ancient because introns are also found at similar positions in homologous genes in some other eukaryotic taxa [3]. Since the taxonomic sampling addressing the prevalence of any of these introns is sparse and because many of these introns are not located in the same phase or even the same relative amino acid position, the actual conservation and ancient nature of these introns requires further verification. The possibility that a subset of spliceosomal introns could be ancestral to the eukaryotic radiation is an exciting one and, if true, suggests that these introns might be useful genomic markers to aid in the elucidation of deep phylogenetic relationships within the domain Eucarya.

Recently, hypotheses describing eukaryotic evolution have coalesced around a limited number of eukaryotic supergroups. One scheme [7] proposes six primary eukaryotic clades: Opisthokonta, Amoebozoa, Plantae, Chromalveolata [8], Rhizaria [9] and Excavata [6,10]. The strength of the evidence supporting each of these supergroups varies, as does the degree of organismal sampling within the proposed assemblages. The relationships among the supergroups themselves are currently undefined: i.e., their relative branching order in the eukaryotic tree is unknown. Even with concatenated data sets com- prising hundreds of individual sequences, the approaches of molecular phylogenetics are increasingly challenged to provide robust and compelling answers to this question.

In this report, we identify an intron in Rpl7a, the gene encoding the G. lamblia ribosomal protein L7a and an additional spliceosomal intron in an unassigned ORF that encodes a non-conserved protein. We observe striking similarities among the G. lamblia, T. vaginalis and C. membranifera introns. At the same time, departures from the sequence constraints within these motifs may discriminate some of the splicing mechanisms employed within these eukaryotic groups. Our extensive examination of the phylogenetic distribution and properties of the Rpl7a intron indicate that it is an ancient spliceosomal intron, and our study also provides preliminary evidence uniting three eukaryotic supergroups (Opisthokonta, Amoebozoa, Excavata) to the exclusion of at least two others (Chromalveolata, Plantae). Our investigation of the distribution of the Rpl7a intron is the most extensive examination to date of a conserved intron position in eukaryotes. Taken together, these results argue that further examination of the patterns of intron conservation and distribution within the eukaryotic domain, like other shared derived characters such as gene fusions, insertions and gene replacements, can provide a valuable adjunct for evaluating proposed phylogenetic groupings and frameworks derived from sequence comparisons.

\section{Results and discussion \\ A conventional spliceosomal intron in the Giardia lamblia Rpl7a gene}

In comparing ribosomal protein L7a homologs from various organisms, we noted that the predicted sequence for the G. lamblia protein [GenBank:EAA41652] appeared abnormally truncated at the C-terminal end relative to other eukaryotic and archaeal sequences, terminating at position 171 of S. cerevisiae L7a (Fig. 1). In addition, this truncated sequence displays unusual divergence after yeast position 161, which is unexpected because this Cterminal region corresponds to a highly conserved portion of L7a. Further examination of the G. lamblia gene sequence revealed a 109-nt intron whose removal results in a predicted L7a protein sequence similar in length to that of other eukaryotic L7a homologs, and that aligns readily with them downstream of position 161 (Fig. 1). The assigned intron boundary sequences, GT...AG (Fig. 2), are those of conventional spliceosomal introns.

The Rpl7a intron is only the second reported spliceosomal-type intron in G. lamblia, a diplomonad widely considered to be a deep-branching eukaryote. The other example (35 nt in length) resides in a putative [2Fe-2S] ferredoxin gene and begins with CT rather than GT at the $5^{\prime}$ intron boundary [1]. Comparison of the sequences of 
S. cerevisiae

H. sapiens

D. melanogaster

A. castellanii

J. Iibera

T.pyriformis

E.gracilis

H.marismortui

G. lamblia

G
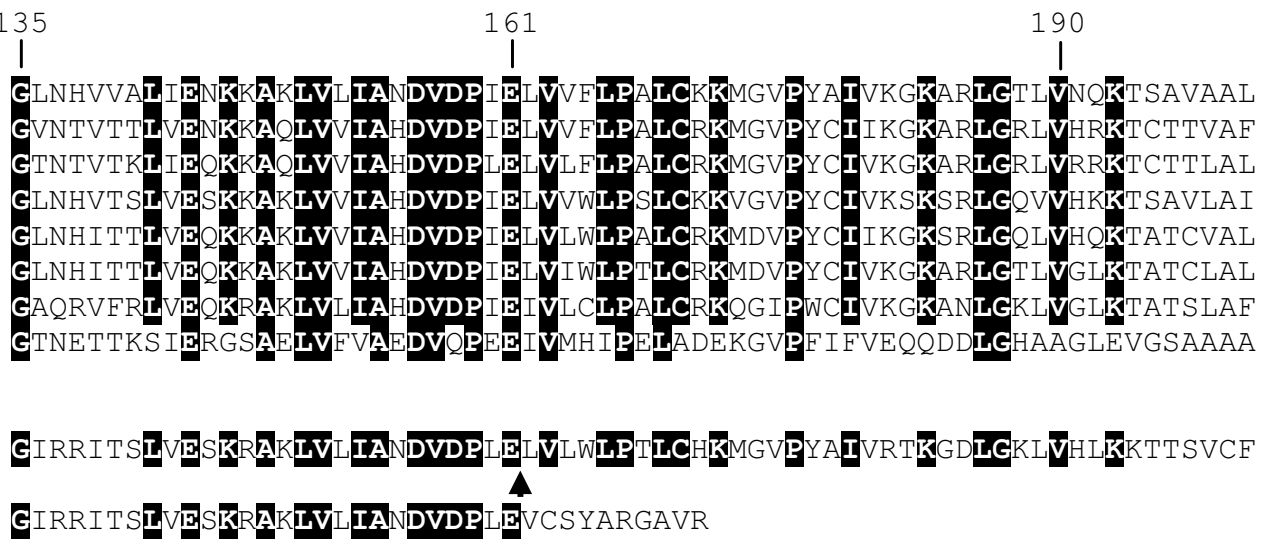

G. Iamblia (EAA41652)

Figure I

Clustal $X$ alignment of ribosomal protein L7a amino acid sequences. The alignment comprises the portion corresponding to positions I 35 to 200 in the Saccharomyces cerevisiae protein (full organism names are listed in Table I). At highly conserved positions at which a single amino acid predominates, residues are indicated as white letters on a black background. The arrowhead denotes the location of a conserved spliceosomal intron. The predicted Giardia lamblia protein sequence either with (above arrowhead) or without (below arrowhead) removal of the intron is shown. Sources of the sequences are: S. cerevisiae [GenBank:AAB65045], Homo sapiens [GenBank:NP 000963], D. melanogaster [GenBank:AAN09172], A. castellanii [GenBank:AY925000], J. libera [GenBank:AY924997], T. pyriformis [GenBank:DQI I8092], E. gracilis [GenBank:], H. marismortui [GenBank:YP |34885] and G. lamblia [GenBank:AACBOI0000I9].

the two G. lamblia introns reveals striking sequence conservation at the intron termini (Fig. 2). Notably, this similarity includes the region predicted to contain the intron branch-point sequence [1] (underlined in Fig. 2). Likewise, both the 5' and 3' intron boundary sequences of the two G. lamblia introns display extensive sequence similarity to the consensus sequence of the known $T$. vaginalis introns (Fig. 2). However, there are some significant nucleotide differences between the G. lamblia Rpl7a intron and the $T$. vaginalis consensus motif that suggest somewhat different sequence requirements for splicing in these two organisms.

As mentioned previously, both CT and GT sequences have now been observed at the 5 ' intron boundary of $G$. lamblia introns. This observation is made even more significant by the demonstration of efficient splicing of the G. lamblia ferredoxin intron in a T. vaginalis in vivo splicing system, but only after changing the CT to a canonical GT at the intron 5' boundary [3]. Our identification of a second G. lamblia intron that contains a canonical GT indicates that the CT sequence is not an absolute requirement for splicing of these introns in G. lamblia and also suggests that the constraints on $5^{\prime}$ intron boundary sequences are different in these two organisms.

The second important observation is derived from a comparison of the sequences of the putative branch-point motifs contained within the two G. lamblia introns. The G. lamblia ferredoxin intron has the putative branch-point sequence ACTAAC, which is identical to that observed in all the identified T. vaginalis introns. The G. lamblia Rpl7a intron instead contains the sequence ACTGAC. By creating site-directed mutants of a T. vaginalis splicing reporter construct using an intron from a poly(A) polymerase gene, Vaňáčová et al. [3] demonstrated the importance of this sequence element and its position relative to the intron 3 ' end for efficient in vivo splicing in T. vaginalis. However, single-nucleotide substitutions at the position corresponding to the $\mathrm{G}$ nucleotide in the branch-point sequence of the G. lamblia Rpl7a intron were not examined for their effects on splicing. Therefore, it is possible that ACTGAC could be a functional branch-point sequence for the splicing of $T$. vaginalis introns.

The majority of the introns identified in the T. vaginalis genome were found using a sequence-pattern search 


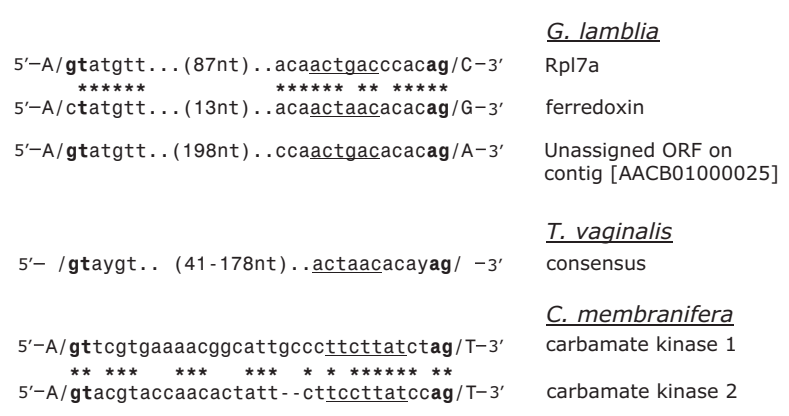

Figure 2

Conservation of intron boundary sequences in Giardia Iamblia, Trichomonas vaginalis and Carpediemonas membranifera. Positions of nucleotide identity within the intron sequences in $G$. lamblia and $C$. membranifera are indicated with asterisks. Potential intron branch-point sequences are underlined. The distance between the intron boundary sequences is indicated for each $G$. lamblia intron as is the distance variation between these sequences seen in the $T$. vaginalis introns used to derive the consensus sequence shown. The ferredoxin intron sequence is from [I], the $T$. vaginalis introns are from [3], and the carbamate kinase intron sequences are from [2].

strategy based primarily on nucleotide conservation observed between the intron boundary sequences of the first identified T. vaginalis intron and the G. lamblia ferredoxin intron. The identified introns, 39 in total, vary in length between 59 and $196 \mathrm{nt}$. They contain a consensus 5 ' splice site sequence GTAYGT and, based on constraints imposed in the search parameters, the 3' splice-site sequence ACTAACACAYAG, where the nucleotides in bold italics indicate the potential branch-point sequence.

To investigate whether an additional group of $T$. vaginalis introns might instead contain variations at the fourth nucleotide position (bold italics) of the branch-point sequence, ACTAAC, the partial $T$. vaginalis genome sequence was searched for potential introns containing these properties. We also incorporated into our search parameters an allowance for other sequence differences within the 3 ' intron boundary sequence, such as those observed when comparing the two G. lamblia introns. Using the pattern-search algorithm PatScan [11], we searched the entire preliminary genomic data (3X coverage) for all sequences containing the sequence pattern [5'- GTAYGT...5-500 nt...ACTBACNCAYAG-3'], where B $=\mathrm{C}, \mathrm{G}$ or $\mathrm{T} ; \mathrm{Y}=\mathrm{C}$ or $\mathrm{T} ; \mathrm{N}=$ any nucleotide and the branchpoint sequence is in bold italics. Remarkably, only two matches to this pattern were found in the entire T. vaginalis genome data set and neither of these sequence patterns appears to be an intron candidate. Furthermore, neither of these two sequences contains ACTGAC in the predicted position for the intron branch-point sequence. This result further emphasizes the importance of the ACTAAC sequence motif in the T. vaginalis introns and the differences in sequence constraints apparent between these introns and those of G. lamblia. If additional T. vaginalis spliceosomal introns are found ( $<519 \mathrm{nt}$ in size) that contain alternative sequences at the 3 ' intron boundary, sequence differences must also be present in the 5 ' intron boundary that prevent the search algorithm from finding these intron candidates using the parameters we have employed.

Using search parameters similar to those above (but allowing $\mathrm{G}$ or $\mathrm{C}$ as the starting nucleotide at the intron $5^{\prime}$ end), we searched the G. lamblia partial genomic data set for intron candidates. As expected, the Rpl7a and ferredoxin introns are detected by this method, as is an additional set of 15 matches to this sequence pattern. Of these matches, only one was a likely intron candidate that had the potential to disrupt a predicted ORF, among other criteria. Since the ORF in which this candidate intron resides (or that it disrupts) does not encode a conserved protein - unlike the Rpl7a intron - further experimentation was required to prove whether or not it is an intron. Removal of the intron candidate sequence, nt positions 1263 to 1482 of the G. lamblia contig [GenBank:AACB01000025], extends the predicted protein encoded by the unassigned ORF [GenBank:EAA41257] by an additional 118 amino acids at its amino terminus. Further, when the intron sequence is removed, the inferred initiation codon is now positioned directly adjacent to an AT-rich motif that Yee et al. [12] have previously identified as a promoter element for other G. lamblia genes. RT-PCR analysis and sequencing of cDNA clones obtained from the corresponding mRNA (see Additional File 1) confirm the existence of this third G. lamblia spliceosomal intron, which is $220 \mathrm{nt}$ long and exhibits intron boundary sequences similar to those of the ferrodoxin and Rpl7a introns (Fig. 2).

Extensive genomic DNA information is not yet available for C. membranifera, and to date only two introns, both small, have been identified in this protist [2]. In these introns, potential branch-point sequences (underlined in Fig. 2) are found abutting the $3^{\prime}$ intron boundary, as is observed in the G. lamblia and T. vaginalis introns. While the G. lamblia introns lack an obvious polypyrimidine tract in the vicinity of the 3 ' end of the intron, C. membranifera introns and the majority of the T. vaginalis introns do exhibit pyrimidine-rich sequences immediately upstream of the 3 ' intron/exon boundary. However, in the C. membranifera introns these pyrimidine-rich sequences include the potential branch-point sequences whereas in T. vaginalis these sequences (usually T-rich) are found upstream 
of the potential branch-point sequences. It is also apparent that the C. membranifera introns exhibit less sequence conservation at their 3 ' intron boundaries than do the $G$. lamblia and T. vaginalis introns. These important differences in the properties apparent in C. membranifera introns are particularly relevant to this comparison of intron structure because this organism appears to have a closer evolutionary relationship to G. lamblia than does $T$. vaginalis [13]. The variety of differences observed in intron structure from each of these three organisms seems to further differentiate the sequence requirements for the splicing mechanisms employed in these eukaryotic groups. The identification of homologs of snRNAs in these organisms may give insight and permit comparisons of novel splicing mechanisms employed in the removal of these introns, characterized by the above-mentioned conserved intronic elements and potential branch-point sequences unusually close to the intron 3 ' end.

\section{Phylogenetic distribution and properties of the Rpl7a intron indicate that it is ancient}

Given the phylogenetic conservation of the L7a protein in the eukaryotic domain, we searched available eukaryotic genomic databases to determine whether an intron at the same position as in G. lamblia is conserved in the Rpl7a gene of other organisms. Somewhat surprisingly, we identified a conventional spliceosomal intron at exactly the same position and in the same phase within Rpl7a in many animal (opisthokont) taxa and in the amoebozoon Dictyostelium discoideum (Fig. 3). This result, in concert with the proposed deep branching position of Giardia, suggested that the Rpl7a intron might be an ancient eukaryotic spliceosomal intron. Accordingly, we undertook a more detailed investigation of the occurrence of this particular intron within the eukaryotic domain. Using EST sequences available through the Protist EST Program (PEP), PCR primers were designed to amplify genomic Rpl7a sequences from a wider array of eukaryotes, particularly those placed in the controversial taxon Excavata [14]. We identified the Rpl7a intron in three additional Amoebozoa representatives spanning amoebozoan diversity [15]. We also found the intron in three additional groups from Excavata: jakobids, malawimonads and Trimastix pyriformis (Fig. 3). The intron is variable in size in these organisms but was only ever found in frame 0 of the coding region. The jakobid Rpl7a introns (541$988 \mathrm{nt}$ ) are the largest characterized to date in this taxon, notably longer than the 156-nt intron found in the J. libera $\beta$-tubulin gene [16]. The Rpl7a intron is also the first reported example of an intron from Trimastix, although other introns have been identified in this organism (A.J. Roger, pers. comm.). Malawimonas jakobiformis appears to have two copies of Rpl7a, each containing the intron. Table 1 summarizes the organisms surveyed for the presence of the Rpl7a intron.

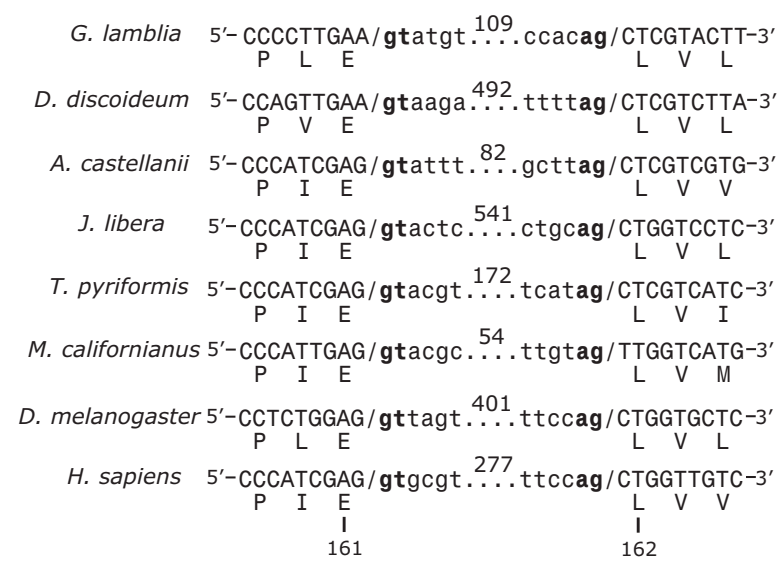

Figure 3

An evolutionarily conserved spliceosomal intron within Rpl7a of representative organisms of the domain Eucarya. Nucleotide sequences at the exon-intron junctions are shown, with the exon sequences in uppercase letters and intron sequences in lowercase. The total length of each intron is shown above the dotted lines. The conventional spliceosomal intronic boundary sequences are bolded (gt...ag). One-letter amino acid abbreviations for the corresponding L7a protein sequences are indicated below the exon sequences. Amino acid position within the L7a protein is indicated at the bottom of the figure and corresponds to the $S$. cerevisiae sequence, as in Fig. [I]. Sequences from $\mathrm{NCBI}$ are: D. discoideum [GenBank:ACII6100], D. melanogaster [GenBank:X82782]; H. sapiens [GenBank:X52138]; and G. lamblia [GenBank:AACBO1000019]. Sequences determined in the present study are: A. castellanii [GenBank:AY925008]; J. libera [GenBank:AY925006]; T. pyriformis [GenBank:AY9250II]; M. californianus [GenBank:AY925003].

Within Opisthokonta and Amoebozoa, several groups appear to lack the Rpl7a intron. Over time introns are randomly lost, so a punctate distribution within established eukaryotic groups is not unexpected. Independent cases of intron loss can be inferred when the relationship among groups is known and the intron is present in a common ancestor. This is the case for taxa from the Opisthokonta and the Entamoebidae (amoebozoons) that are missing the Rpl7a intron. The Rpl7a intron is present in several amoebozoons that branch outside $[15,17]$ of those that lack the intron. A particularly interesting case of predicted intron loss is the Anopheles gambiae Rpl7a [GenBank:AAAB01008960], which lacks any introns. This situation is in stark contrast to other hexapod Rpl7a sequences, which contain introns in addition to the conserved intron discussed here. The A. gambiae case may be an example of intron loss mediated by a reverse tran- 
Table I: Organisms surveyed for the presence of the Rpl7a intron

\begin{tabular}{lll}
\hline Organism $^{1}$ & Number $^{2}$ & Intron Size (nt) \\
\hline
\end{tabular}

\section{With intron:}

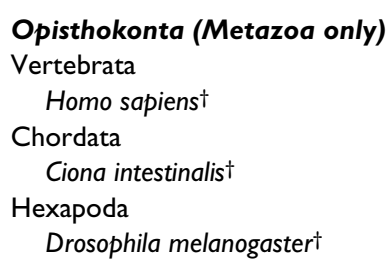

\section{Amoebozoa}

Dictyostelium discoideum ${ }^{\dagger}$ Physarum polycephalum ${ }^{* \dagger}$ Acanthamoeba castellanii*† Hartmannella vermiformis*†

\section{Excavata \\ Jakobidae \\ Jakoba libera*t \\ Reclinomonas americana*t \\ Seculamonas ecuadoriensis*t \\ Trimastix (Trimastix pyriformis*†) \\ Malawimonadidae \\ Malawimonas jakobiformis*t \\ Malawimonas californianus*t \\ Diplomonadida (Giardia lamblia)}

\section{Without intron:}

Chromalveolata

Alveolata

Heterokonta (stramenopiles)

\section{Plantae}

Streptophyta

Rhodophyta

Chlorophyta

\section{Rhizaria}

Cercozoa (Bigelowiella natans*)

\section{Opisthokonta}

Fungi

Nematoda/Trematoda

Hexapoda

Capsaspora owczarzaki*

\section{Amoebozoa}

Entamoebidae

\section{Excavata}

Heterolobosea (Naegleria gruberi*)

Euglenozoa

Trypanosomatidae

Euglenida (Euglena gracilis*)
Table I: Organisms surveyed for the presence of the Rpl7a intron (Continued)

Parabasalia (Trichomonas vaginalis)

Diplomonadida (Spironucleus barkhanus*)

IAn asterisk $(*)$ denotes organisms for which PCR amplification of the $R p / 7 a$ gene was performed; $\dagger$ indicates that expressed sequence tag (EST) data exist that confirm the absence of the intron in the corresponding mRNA.

${ }^{2}$ Numbers in brackets refer to the number of organisms examined within each group; a complete list is provided in Additional File 3. ${ }^{3}$ Two versions of the Rpl7a gene exist in this organism, containing introns of slightly different length.

scription mechanism $[18,19]$ and is also consistent with observed intron loss patterns in the hexapods [20].

None of the examined members of Plantae, Chromalveolata or Rhizaria contained the conserved Rpl7a intron. Only a single member of Rhizaria (B. natans) was investigated here so it is premature to conclude that the Rpl7a intron is absent altogether from this supergroup. Our survey failed to detect the Rpl7a intron in representatives of the Euglenozoa, Heterolobosea or Parabasalia, all eukaryotic groups for which inclusion in a larger Excavata assemblage is only weakly supported [6]. Whereas emerging data suggest that $E$. gracilis is well endowed with spliceosomal introns [21-23], intron distribution appears to be sparse in heteroloboseans [24]. The diplomonad Spironucleus barkhanus does not have the Rpl7a intron, likely a result of intron loss in this organism given the presence of the intron in other related Excavata.

We considered other (less probable) explanations that the phylogenetic distribution of the Rpl7a intron could be explained by independent events of intron gain or by lateral gene transfers. We note that the Rpl7a exon boundary sequences conform poorly to the conserved proto-splice site sequence (A,C)AG/G [25] (Fig. 1 and 3). The predicted amino acids flanking the intron insertion site are highly conserved, including in the archaeal homologs, resulting in a functional constraint on the DNA sequence abutting the intron. This observation argues against a distribution of the Rpl7a intron resulting from multiple intron gains at proto-splice sites. Supporting the argument against intron gain is the apparent very low density of introns in G. lamblia. This is also in agreement with recent data suggesting that few shared intron positions between distantly related taxa are due to parallel gain (i.e., independent insertion) at proto-splice sites [25].

The additional possibility exists that the phylogenetic distribution of the intron, particularly its presence in G. lamblia, could reflect eukaryote-to-eukaryote lateral gene transfer events. A phylogenetic analysis (see Additional 
File 2) of L7a protein sequences from 20 representative eukaryotes and two archaeons does not indicate an obvious unexpected affinity of $G$. lamblia with any other eukaryotic group. Cumulatively, the above observations argue that the Rpl7a intron is ancestral to many eukaryotic groupings and has been lost sporadically in various eukaryotic taxa, in which case the Rpl7a intron would be one of the oldest introns found to date.

\section{Phylogenetic implications}

Depending on one's views of the eukaryotic tree, there are two possibilities to explain the observed distribution of the Rpl7 $a$ intron with regard to intron loss. In the context of more recent proposals of a basally unresolved eukaryotic tree $[7,26]$, the Rpl7a intron would have to have been lost multiple times at the base of Plantae, Chromalveolata and (tentatively) Rhizaria, but maintained in Opisthokonta, Amoebozoa and Excavata. Alternatively, if one assumes a specific relationship among Opisthokonta, Amoebozoa and Excavata, it is only necessary to invoke a single loss of the Rpl7a intron in a common ancestor of Plantae/Chromalveolata/Rhizaria, to explain the apparent absence of this intron in these three supergroups.

While multiple cases of intron gain seem unlikely (as discussed above), a single intron gain at the base of Opisthokonta, Amoebozoa and Excavata would result in the observed distribution, assuming the Plantae/Chromalveolata/Rhizaria had already diverged. While additional intermediate possibilities exist with regards to various intron loss and/or gain events, we propose that a single loss or gain is the most parsimonious explanation for the observed distribution of the Rpl7a intron, supporting a grouping of Opisthokonta, Amoebozoa and Excavata.

Although the distribution of the Rpl7a intron does not position the root of the eukaryotic tree, it may help to resolve some of the basal branches. It is important to note that Excavata is a tenuous grouping that may in fact be polyphyletic: thus, members of Excavata without the intron may fall on either side of a putative loss/gain event. In this instance, it is possible that one or more members of Excavata will be found to group with the Plantae/Chromalveolata/Rhizaria consortium. Conversely, if additional evidence robustly groups the organisms in question within Excavata, this result would imply that they lost the intron independently, as is evidently the case for Fungi within Opisthokonta.

New eukaryotic genome sequences may well reveal the Rpl7a intron in other representatives of Plantae, Chromalveolata and/or (particularly) Rhizaria than those listed in Table 1. Although such a finding would necessarily require reinterpretation of some of the conclusions reached here, discovery of the Rpl7a intron in these other eukaryotic supergroups would only strengthen the argument that this intron is indeed ancient. At present, the simplest explanation for the observed distribution of the Rpl7a intron is a specific relationship uniting Opisthokonta, Amoebozoa and Excavata to the exclusion of Plantae, Chromalveolata and possibly Rhizaria. However, additional characters will need to be found in order to strengthen this proposed assemblage.

\section{Conclusion}

The properties possessed by the first identified G. lamblia spliceosomal-type intron (35 nt in length) raised questions regarding the importance of its non-canonical 5' intron boundary and possible constraints on the size of Giardia introns. Furthermore, it was clear that the identification of additional spliceosomal introns would be required to assess the degree of conservation of predicted functional sequence elements within these introns [27]. In this study we have identified in G. lamblia two larger introns (109 and $220 \mathrm{nt}$ ) in genes encoding, respectively, L7a and a non-conserved, unassigned protein. Both of these newly identified introns exhibit a canonical GT 5' intron boundary. Evolutionary conservation of the exact position and phase of the Rpl7a intron (within a highly conserved region of the L7a protein sequence in members of diverse eukaryotic groups) indicates that this particular intron was likely present in the ancestor of these lineages. The Rpl7a intron exhibits all of the hallmark features of an ancient intron, such as widespread distribution, phase 0 positioning, location in an ancient eukaryotic gene, and lack of a proto-splice site sequence; thus, this intron can be considered to be a meaningful phylogenetic marker. Currently, no singular genomic marker definitively resolves the branching order of the eukaryotic supergroups. We propose that patterns of conservation of ancient introns, when larger data sets are examined, may provide such information.

\section{Methods \\ Genomic DNA}

Samples of genomic DNA from various protists (full organism names are listed in Table 1) were kindly provided by B.F. Lang (R. americana, ATCC 50394; S. ecuadoriensis, ATCC 50688; M. jakobiformis, ATCC 50310; M. californianus, ATCC 50740); A.J. Roger (S. barkhanus strain NOR-1A, ATCC 50380+; C. owczarzaki, ATCC 30864; T. pyriformis, ATCC 50562; N. gruberi, ATCC 30224); A.J. Lohan (A. castellanii, ATCC 30010; H. vermiformis, ATCC 50236); D.F. Spencer (E. gracilis strain Z); and J.M. Archibald (B. natans, CCMP 621). Genomic DNA from $J$. libera (ATCC 50422) and P. polycephalum (strain $\mathrm{M}_{3} \mathrm{C}$ ) was obtained by lysing cells in 1\% SDS followed by phenol extraction and ethanol precipitation. 


\section{Characterization of $\mathrm{Rp}$ I7a sequences}

Polymerase chain reaction (PCR) was used to amplify Rpl7a from genomic DNA (100-200 ng), using Invitrogen Taq DNA polymerase. PCR primers used are listed in Additional file 3: Supplemental Tables. PCR cycling conditions were: $3 \mathrm{~min}$ at $95^{\circ} \mathrm{C} ; 35$ cycles of $30 \mathrm{sec}$ at $95^{\circ} \mathrm{C}$, $30 \mathrm{sec}$ at $55^{\circ}, 1 \mathrm{~min}$ at $72^{\circ} \mathrm{C}$; and $10 \mathrm{~min}$ at $72^{\circ} \mathrm{C}$. PCR product bands were isolated from gels using the Sephaglas $^{\mathrm{TM}}$ BandPrep Kit (Amersham Pharmacia Biotech) and the recovered DNA was cloned into the $\mathrm{pCR}^{\circledR} 2.1$ TOPO $^{\circledR}$ vector using the TOPO TA Cloning ${ }^{\circledR}$ Kit (Invitrogen). DNA sequencing was performed using an automated ABI Prism 377 DNA sequencer.

\section{Computer analyses}

Ribosomal protein L7a gene and protein sequences were identified by searching relevant sequence databases using TBLASTN or BLASTP with the $S$. cerevisiae sequences as queries. Protein alignments were generated with ClustalX 1.82 [28] applying default alignment parameters. DNASIS V 2.5 was used for translation of DNA sequences and to assist in the identification of introns within Rpl7a sequences. Searches for spliceosomal-like introns within the preliminary genome sequence data of T. vaginalis and G. lamblia were performed using the sequence pattern search program PatScan [11], recompiled with the constant 'MAX_SEQ-LEN' redefined to '100000000'.

\section{Authors' contributions}

AGR analyzed the L7a protein sequences, discovered the G. lamblia Rpl7a intron, performed genomic searches for additional introns in G. lamblia and T. vaginalis and carried out the RT-PCR experiments to confirm the presence of the third G. lamblia spliceosomal intron. TES examined the phylogenetic conservation of the Rpl7a intron in currently available eukaryotic genome databases, performed all the PCR experiments to determine the prevalence of the intron, and participated in the genomic searches for additional G. lamblia introns. RFW performed the phylogenetic analysis of L7a sequences. MWG made substantial intellectual contributions to this work. All authors participated in the assembly and editing of the manuscript. All authors read and approved the final manuscript.

\section{Additional material}

\section{Additional File 1}

Evidence for a spliceosomal intron in an unassigned G. lamblia ORF. This file (PDF format) presents gel electrophoretic data documenting the results of PCR and RT-PCR experiments to confirm the existence of a putative spliceosomal intron in a non-conserved, unassigned ORF in G. lamblia, as described in the text.

Click here for file

[http://www.biomedcentral.com/content/supplementary/14712148-5-45-S1.pdf]

\section{Additional File 3}

Supplemental Tables. This file (PDF format) contains two tables. Table 1 contains the sequences of the oligonucleotide primers used for amplifying Rpl7a sequences from various eukaryotic taxa. Table 2 is a complete list of all the organisms and sequence sources that were examined for the presence of the Rpl7a intron.

Click here for file

[http://www.biomedcentral.com/content/supplementary/14712148-5-45-S3.pdf]

\section{Additional File 2}

L7aTree. This file (PDF format) presents a maximum likelihood tree generated using L7 a sequences from representative eukaryotic and archaeal taxa. Also included are the methods used to generate the tree, the sources of the sequences and the alignment that was used.

Click here for file

[http://www.biomedcentral.com/content/supplementary/14712148-5-45-S2.pdf]

\section{Acknowledgements}

We are grateful to B.F. Lang (Université de Montréal) and A.J. Roger, A.J. Lohan, D.F. Spencer and J.M. Archibald (Dalhousie University) for generous gifts of genomic DNA. We thank the following members (past and present) of the Roger laboratory for providing Rpl7a EST sequence data: J. Silberman and M. Baumgartner (T. pyriformis), A. Sjögren and E. Gill (N. gruberi), J. Andersson (S. barkhaus), A.J. Roger (C. owczarzaki). Additional Rpl7a EST sequences were provided by P.J. Keeling, University of British Columbia ( $B$. natans) and B.F. Lang (M. californianus, M. jakobiformis, J. libera, R. americana, $S$. ecuadoriensis). We also thank J. Yee (Trent University) for generously providing $G$. lamblia total RNA and for insightful discussions regarding promoter elements; J. Gott (Case Western Reserve University) for helpful discussions; D.F. Spencer for assistance with the PatScan program; and M. Dlutek and A. Fong for performing DNA sequencing. Preliminary sequence data for Trichomonas vaginalis were obtained from The Institute for Genomic Research website at http://www.tigr.org Sequencing of the T. vaginalis genome was funded by the National Institute of Allergy and Infectious Diseases (NIAID). This work was supported by funding to MWG from the Canadian Institutes of Health Research (operating grant MOP-4124) and Genome Canada through Genome Atlantic and the Atlantic Innovation Fund (Protist EST Program). MWG is pleased to acknowledge salary support from the Canadian Research Chairs Program and the Canadian Institute for Advanced Research.

\section{References}

I. Nixon JE, Wang A, Morrison HG, McArthur AG, Sogin ML, Loftus BJ, Samuelson J: A spliceosomal intron in Giardia lamblia. Proc Natl Acad Sci USA 2002, 99:3701-3705.

2. Simpson AG, MacQuarrie EK, Roger AJ: Eukaryotic evolution: early origin of canonical introns. Nature 2002, 41 9:270.

3. Vaňáčová S, Yan W, Carlton JM, Johnson PJ: Spliceosomal introns in the deep-branching eukaryote Trichomonas vaginalis. Proc Natl Acad Sci USA 2005, I 02:4430-4435.

4. Fast NM, Doolittle WF: Trichomonas vaginalis possesses a gene encoding the essential spliceosomal component, PRP8. Mol Biochem Parasitol 1999, 99:275-278.

5. Collins L, Penny D: Complex spliceosomal organization ancestral to extant eukaryotes. Mol Biol Evol 2005, 22:1053-1066.

6. Simpson AG: Cytoskeletal organization, phylogenetic affinities and systematics in the contentious taxon Excavata (Eukaryota). Int J Syst Evol Microbiol 2003, 53:1759-1777.

7. Simpson AG, Roger AJ: The real 'kingdoms' of eukaryotes. Curr Biol 2004, I 4:R693-R696. 
8. Cavalier-Smith T: A revised six-kingdom system of life. Biol Rev Camb Philos Soc 1998, 73:203-266.

9. Nikolaev SI, Berney C, Fahrni JF, Bolivar I, Polet S, Mylnikov AP, Aleshin VV, Petrov NB, Pawlowski J: The twilight of Heliozoa and rise of Rhizaria, an emerging supergroup of amoeboid eukaryotes. Proc Natl Acad Sci USA 2004, I01:8066-8071

10. Simpson AGB, Roger AJ: Excavata and the origin of amitochondriate eukaryotes. In Organelles, Genomes, and Eukaryote Phylogeny: An Evolutionary Synthesis in the Age of Genomics Edited by: Hirt RP, Horner DS. Boca Raton, FL, CRC Press; 2004:27-54.

II. Dsouza M, Larsen N, Overbeek R: Searching for patterns in genomic data. Trends Genet 1997, 13:497-498.

12. Yee J, Mowatt MR, Dennis PP, Nash TE: Transcriptional analysis of the glutamate dehydrogenase gene in the primitive eukaryote, Giardia lamblia. Identification of a primordial gene promoter. J Biol Chem 2000, 275: I |432-I I 439.

13. Simpson AGB, Roger AJ, Silberman JD, Leipe DD, Edgcomb VP, Jermiin LS, Patterson DJ, Sogin ML: Evolutionary history of "earlydiverging" eukaryotes: the excavate taxon Carpediemonas is a close relative of Giardia. Mol Biol Evol 2002, 19:1782-I79|.

14. Cavalier-Smith T: The phagotrophic origin of eukaryotes and phylogenetic classification of Protozoa. Int J Syst Evol Microbiol 2002, 52:297-354

15. Fahrni JF, Bolivar I, Berney C, Nassonova E, Smirnov A, Pawlowski J: Phylogeny of lobose amoebae based on actin and small-subunit ribosomal RNA genes. Mol Biol Evol 2003, 20: I88I- 1886.

16. Archibald JM, O'Kelly CJ, Doolittle WF: The chaperonin genes of jakobid and jakobid-like flagellates: implications for eukaryotic evolution. Mol Biol Evol 2002, 19:422-43I.

17. Baldauf SL, Roger AJ, Wenk-Siefert I, Doolittle WF: A kingdomlevel phylogeny of eukaryotes based on combined protein data. Science 2000, 290:972-977.

18. Roy SW, Gilbert W: The pattern of intron loss. Proc Natl Acad Sci USA 2005, 102:7|3-7|8.

19. Sverdlov AV, Babenko VN, Rogozin IB, Koonin EV: Preferential loss and gain of introns in $3^{\prime}$ portions of genes suggests a reversetranscription mechanism of intron insertion. Gene 2004, 338:85-91.

20. Zdobnov EM, von Mering C, Letunic I, Torrents D, Suyama M, Copley RR, Christophides GK, Thomasova D, Holt RA, Subramanian GM, Mueller H-M, Dimopoulos G, Law JH, Wells MA, Birney E, Charlab R, Halpern AL, Kokoza E, Kraft CL, Lai Z, Lewis S, Louis C, Barillas-Mury C, Nusskern D, Rubin GM, Salzberg SL, Sutton GG, Topalis P, Wides R, Wincker P, Yandell M, Collins FH, Ribeiro J, Gelbart WM, Kafatos FC, Bork P: Comparative genome and proteome analysis of Anopheles gambiae and Drosophila melanogaster. Science 2002, 298:149-159.

21. Breckenridge DG, Watanabe Y-I, Greenwood SJ, Gray MW, Schnare $\mathrm{MN}$ : UI small nuclear RNA and spliceosomal introns in Euglena gracilis. Proc Natl Acad Sci USA 1999, 96:852-856.

22. Canaday J, Tessier LH, Imbault P, Paulus F: Analysis of Euglena gracilis alpha-, beta- and gamma-tubulin genes: introns and premRNA maturation. Mol Genet Genomics 200I, 265:153-160.

23. Russell AG, Watanabe $Y-i$, Charette JM, Gray MW: Unusual features of fibrillarin cDNA and gene structure in Euglena gracilis: evolutionary conservation of core proteins and structural predictions for methylation-guide box C/D snoRNPs throughout the domain Eucarya. Nucleic Acids Res 2005, 33:278|-279|.

24. Logsdon JM Jr: The recent origins of spliceosomal introns revisited. Curr Opin Genet Dev 1998, 8:637-648.

25. Sverdlov AV, Rogozin IB, Babenko VN, Koonin EV: Conservation versus parallel gains in intron evolution. Nucleic Acids Res 2005, 33: $174 \mid-1748$.

26. Baldauf SL: The deep roots of eukaryotes. Science 2003, 300: $1703-1706$.

27. Johnson PJ: Spliceosomal introns in a deep-branching eukaryote: The splice of life. Proc Natl Acad Sci USA 2002, 99:3359-336I.

28. Chenna R, Sugawara H, Koike T, Lopez R, Gibson TJ, Higgins DG, Thompson JD: Multiple sequence alignment with the Clustal series of programs. Nucleic Acids Res 2003, 3 I:3497-3500.
Publish with Bio Med Central and every scientist can read your work free of charge

"BioMed Central will be the most significant development for disseminating the results of biomedical research in our lifetime. "

Sir Paul Nurse, Cancer Research UK

Your research papers will be:

- available free of charge to the entire biomedical community

- peer reviewed and published immediately upon acceptance

- cited in PubMed and archived on PubMed Central

- yours - you keep the copyright

Submit your manuscript here:

http://www.biomedcentral.com/info/publishing_adv.asp
Biomedcentral 